\title{
A $1 \times 4$ power-splitter based on photonic crystal Y-splitter and directional couplers
}

\author{
Fulya Bagci, Baris Akaoglu( ${ }^{*}$ \\ Department of Engineering Physics, Faculty of Engineering, Ankara University, Turkey. \\ (*) Email: akaoglu@eng.ankara.edu.tr \\ Received / Recibido: 02/04/2013. Revised / Revisado: 16/07/2013. Accepted / Aceptado: 04/09/2013. \\ DOI: http://dx.doi.org/10.7149/OPA.46.3.265
}

\begin{abstract}
:
A $1 \times 4$ photonic crystal power splitter operating in the optical communication window is demonstrated by plane-wave expansion and finite-difference time-domain simulations. The device consists of one Y-junction and two directional couplers. Double $60^{\circ}$ bends are formed at the exit of directional couplers to prevent crosstalk. The Y-junction, double bends and coupling holes at the directional couplers are optimized to increase the transmission efficiency at the output ports. The simulation results exhibit almost equal power splitting with low loss characteristic. The investigated design shows potential for optical switching and routing applications in optical communications.
\end{abstract}

Key words: Photonic Crystal Waveguide, Y-Junction, Directional Coupler, Power-Splitter, FiniteDifference Time-Domain Method.

\section{REFERENCES AND LINKS / REFERENCIAS Y ENLACES}

[1]. M. Djavid, A. Ghaffari, F. Monifi, M. S. Abrishamian, "Heterostructure photonic crystal channel drop filters using mirror cavities", J. Opt. A: Pure Appl. Opt. 10, 055203 (2008). DOI

[2]. A. Martinez, P. Sanchis, J. Marti, "Mach-Zehnder interferometers in photonic crystals", Opt. Quantum Electron. 37, 77-93 (2005). DOI

[3]. S. Haxha, W. Belhadj, F. Abdelmalek, H. Bouchriha, "Analysis of wavelength demultiplexer based on photonic crystals", Proc. Inst. Elect. Eng. Optoelectron. 152, 193-198 (2005). DOI

[4]. I. Park, H.-S. Lee, H.-J. Kim, K.-M. Moon, S.-G. Lee, B.-H. O, S.-G. Park, E.-H. Lee, "Photonic crystal powersplitter based on directional coupling", Opt. Express 12, 3599-3604 (2004). DOI

[5]. A. Ghaffari, M. Djavid, F. Monifi, M. S. Abrishamian, "Photonic crystal power splitter and wavelength multi/demultiplexer based on directional coupling", J. Opt. A:Pure Appl. Opt. 10, 075203 (2008). DOI

[6]. A. Mekis, J. C. Chen, I. Kurland, S. Fan, P. R. Villeneuve, J. D. Joannopoulos, "High transmission through sharp bends in photonic crystal waveguides", Phys. Rev. Lett. 77, 18, 3787-3790 (1996). DOI

[7]. T. Trifonov, L. F. Marsal, A. Rodríguez, J. Pallarès, R. Alcubilla, "Effects of symmetry reduction in twodimensional square and triangular lattices", Phys. Rev. B 69, 235112-1-11 (2004). DOI

[8]. S. Noda, T. Baba, Roadmap on Photonic Crystals, page 255, Springer, New York (2003). DOI

[9]. K. B. Chung, J. S. Yoon, "Properties of a $1 \times 4$ optical power splitter made of photonic crystal waveguides", Opt. Quantum Electron. 35, 955-966 (2003). DOI

[10]. R. Wilson, T. J. Karle, I. Moerman, T. F Krauss, "Efficient photonic crystal Y-junctions", J. Opt. A: Pure Appl. Opt. 5, S76-S80 (2003). DOI

[11]. L. O'Faolain, X. Yuan, D. McIntyre, S. Thoms, H. Chong, R. M. De la Rue, T. F. Krauss, "Low-loss propagation in photonic crystal Waveguides", Electron. Lett. 42, 1454-1455 (2006). DOI

[12]. M. Qui, "Effective index method for heterostructure-slab-waveguide-based two dimensional photonic crystals", Appl. Phys. Lett. 81, 1163-1166 (2002). DOI

[13]. PhotonDesign, CrystalWave, http://www.photond.com/ retrieved 06.03.2012.

[14]. J. Zimmermann, M. Kamp, A. Forchel, R. Marz, "Photonic crystal waveguide directional couplers as wavelength selective filters", Opt. Commun. 230, 387-392 (2004). DOI 
[15].H. Badaoui, M. Feham, M. Abri, "Double bends and Y-shaped splitter design for integrated optics", Prog. Electromagn. Res. Lett. 28, 129-138 (2012). DOI

[16].Z. Chang-Zhu, L. Ya-Zhao, L. Zhi-Yuan, "Waveguide bend of $90^{\circ}$ in two-dimensional triangular lattice silicon photonic crystal slabs", Chin. Phys. Lett. 27, 084203 (2010). DOI

[17]. L. J. Kauppinen, T. J. Pinkert, H. J. W. M. Hoekstra, R. M. de Ridder, "Photonic crystal cavity based Ysplitter for the mechano-optical switching", IEEE Photon. Technol. L. 22, 13, 966-968 (2010). DOI

[18].E. P. Kosmidou, E. E. Kriezis, T. D. Tsiboukis, "Analysis of tunable photonic crystal directional couplers", J. Appl. Phys. 100, 043118 (2006). DOI

[19]. A.Ghaffari, M. Djvaid, F. Monifi, M. S. Abrishamian, "Power splitters with different output power levels based on directional coupling", PIERS Proceeedings, pp. 678-680, USA (2008).

[20].Y. Tanaka, H. Nakamura, Y. Sugimoto, N. Ikeda, K. Asakawa, K. Inoue, "Coupling properties in a 2-D photonic crystal slab directional coupler with a triangular lattice of air holes", IEEE J. Quant. Electron. 41, 76-84 (2005). DOI

[21]. A. Martinez, F. Cuesta, A. Griol, D. Mira, J. Garcia, "Photonic-crystal 180 power splitter based on coupled-cavity waveguides", Appl. Phys. Lett. 83, 3033-3035 (2003). DOI

\section{Introduction}

Photonic crystals (PCs) are periodic dielectric/metallic arrangements that are capable of manipulating the propagation of light with their unique photonic band gap (PBG) property. Frequencies that fall inside the PBG are prohibited to propagate inside the crystal. By using the strong confinement of light by the PBG, various types of optoelectronic devices, such as channel-drop filters [1], Mach-Zehnder interferometers [2], wavelength division multiplexers [3] and power splitters [4,5] can be designed.

PCs can be realized in two different schemes as dielectric rods in air or air holes etched into a dielectric medium. The advantage of the first scheme is that waveguides created by removing a row of holes are single-moded. Therefore, as opposed to "holes in dielectric" scheme, higher order modes cannot be excited at discontunities, such as bends. Highly efficient $90^{\circ}$ bends can be designed in these systems. For example, a $90^{\circ}$ waveguide bend with $95 \%$ transmission is reported in square array of dielectric rods [6]. However, "rods in air" scheme does not provide sufficient vertical confinement as "holes in dielectric" scheme and its practical feasibility into optical devices is difficult. In "holes in dielectric" scheme, light can be guided through the line-defect by PBG effect and in the vertical dimension by total internal reflection. It is well known that among different lattice types, triangular lattice of air holes in dielectric medium provide the largest TE PBG [7].
Therefore, we choose the investigated PC structure as triangular symmetry of air holes in Si slab.

The dimensions of the optoelectronic devices can be adjusted for a minimum size of the desired optical functionality by using PC bend waveguides. It is possible to realize low loss bends in PC platforms. Usually square lattice is used to create a bend of $90^{\circ}$ and triangular lattice is used to create a bend of $60^{\circ}$ due to the direction of primitive lattice vectors [8]. Tbranches are generally formed in square lattice of dielectric rods and Y-branches are formed in triangular lattice of holes [9]. For example, a $1 \times 4$ power splitter is designed on a square lattice of dielectric rods by combining three $\mathrm{T}$-junctions with $90^{\circ}$ bends [9].

An ideal bend must have a high transmission and low dispersion in a large bandwidth range. Bends are essential in optical power splitters, which are main building blocks of optical integrated circuits. Ideally, a $\mathrm{N} \times$ power splitter should divide the input power to $\mathrm{N}$ equal powers with almost zero reflection. However, the straightforward $\mathrm{T}$-junctions and $\mathrm{Y}$-junctions tend to be multi-moded and mode-mixing problems arise at the intersections. The multimode effects can be eliminated by adding smaller holes at the bending core and hence the transmission efficiency can be highly improved [10]. The transmission bandwidth of $1 \times 4$ power splitter is improved by adding two small holes with 0.07 a radius between the input and output of T-branches [9]. Alternatively, power splitting 
can be performed by using PC based directional couplers which have much shorter beat lengths compared to the conventional ones. Here we demonstrate an efficient $1 \times 4$ power splitter based on a PC Y-junction and directional couplers which divide the input power into four parts in the optical communication window.

\section{Design and numerical calculations}

The PC based power splitter designed in this study is depicted in Fig. 1. The splitter is based on a PC based Y-junction and directional couplers. The PC suspended membrane structure consists of a triangular array of holes with $130 \mathrm{~nm}$ radius $(0.3 a)$ in a Si slab of height $220 \mathrm{~nm}$. The lattice constant, a is adjusted to $432.5 \mathrm{~nm}$. Membrane slab structures can be fabricated by selectively etching the substrate beneath the PC regions forming an air-bridge [11]. This structure provides a symmetric configuration preventing the decoupling of orthogonal polarisation states and a stronger index confinement along the vertical dimension. Waveguides are formed by omitting a line of holes. The Y-junction is formed by intersecting three waveguides with a $120^{\circ}$ joint at the angular section. The directional couplers are constructed by omitting a line of holes parallel to the arms of the Y-junction. Power-splitting based on directional coupling is realized by calculating the coupling length for equal power transfer. In order to reduce the crosstalk between output ports and exit light parallel to entrance, double $60^{\circ}$ bend waveguides are used at the end of PC directional couplers.

Three-dimensional calculations are realized in two-dimensions by using an effective index value of 2.75 [12]. The band diagrams are obtained by using plane expansion method of the Band Solver tool of the commercial software package CrystalWave with a grid spacing of $(a / 16, a / 128)[13]$ and the transmission spectra are obtained by finite-difference time-domain calculations with a grid spacing of $a / 32$ by using CrystalWave [13]. The FDTD calculation is run until all the light has disappeared from the PC and the convergence of the results is checked under different simulation time and grid spacing

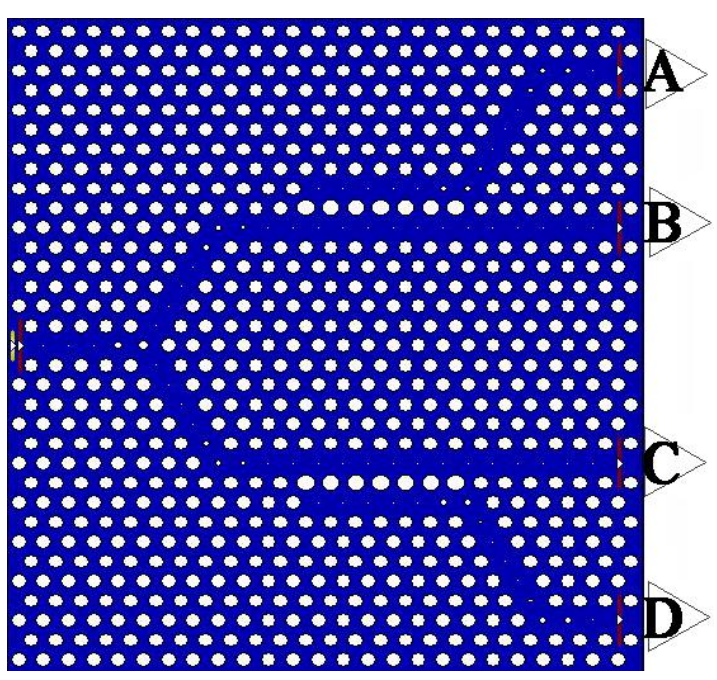

Fig. 1. The designed photonic crystal based power-splitter structure. The yellow line is the source and the red lines are detectors.

values. The thickness of the perfectly matched boundary layer is selected to be one lattice constant and along four sides of the simulation environment. In the finite-difference timedomain simulations, a TE-polarized Gaussian wave with a wide bandwidth centred at $1.55 \mu \mathrm{m}$ is launched at the input entrance to simulate the wave propagation in the structure. The transmission spectra are obtained by normalizing the intensity at the output sensor with respect to the reference sensor at the input entrance. The optimisation of hole radii is realized by using the Kallistos optimisaton tool of CrystalWave [13]. One independent variable as the radius of the included hole is introduced to the optimisation tool for the $60^{\circ}$ bend design and two independent variables as the radii of two holes at the junction are introduced to the optimisation tool for the Y-junction design. The optimisation problem is defined as "global" and the progress of global optimisation is viewed with the "tree view monitoring interface". It is ensured that the best result lies on the longest branch where the optimiser tried more variations in this parameter region and more likely to catch a global maximum. The optimisation is carried out for the wavelength range of $1.5 \mu \mathrm{m}$ and $1.6 \mu \mathrm{m}$. The maximum function evaluations are set to 50 so that the optimisation process with FDTD runs lasts until the number of iterations is completed. The Band 


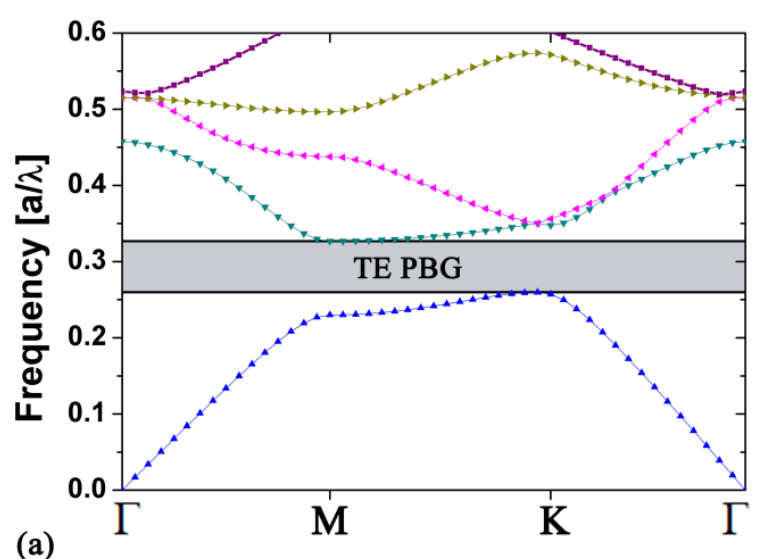

(a)

Fig. 2(a) Band structure for a photonic crystal comprising a triangular lattice of holes with $r=0.30 \mathrm{a}$ and $h=220 \mathrm{~nm}$, (b) dispersion of waveguide modes of the photonic crystal waveguide.

(b)

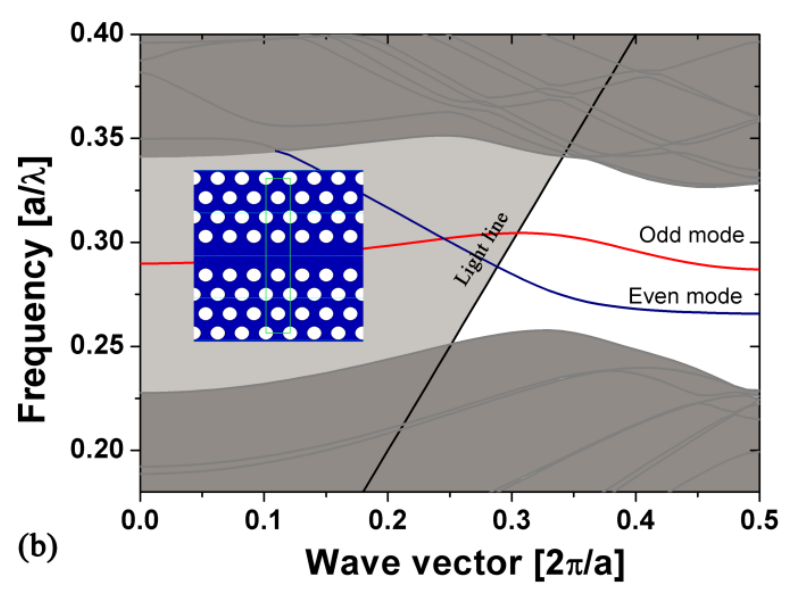

In the wavelength range between $1.535 \mu \mathrm{m}$ and $1.545 \mu \mathrm{m}$, and between $1.552 \mu \mathrm{m}$ and $1.567 \mu \mathrm{m}$, the transmission remains over $70 \%$. Maximum transmission is obtained as $77.4 \%$ at $1.562 \mu \mathrm{m}$ $(0.277(a / \lambda))$.

One degree of freedom as the radius of the included hole is taken into account for each of the geometrical configurations shown in Fig. 3 and the optimisations are performed by using global optimisation algorithm. Each time one independent variable as the radius of the included hole is introduced to the optimisation algorithm with its initial value and upper and lower bounds. The flux detected at the output sensor relative to the flux through the input sensor is aimed to be maximized. It is noteworthy to mention that identical holes are placed for the two bends to maintain symmetry.

At first, one hole is added in the bending core and the optimum radius is found as $0.12 a$ (Fig. 3(b)). The transmission spectrum is observed to be highly broadened with $38 \mathrm{~nm}$ bandwidth (T>70\%) (Fig. 4(b)). However, the transmission curve exhibits fluctuations. In configuration (c), a hole is added in the left side of configuration (b) and the optimum radius is found as $0.125 a$ (Fig. 3(c)). At this case, the transmission is dropped below $60 \%$ in the spectral range between $1.52 \mu \mathrm{m}$ and $1.56 \mu \mathrm{m}$ (Fig. 4(c)). For the case where the hole in the core center is removed and an optimised hole with a radius of $0.08 a$ is placed at the right side 


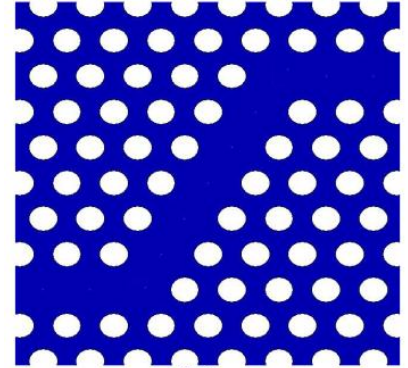

(a)

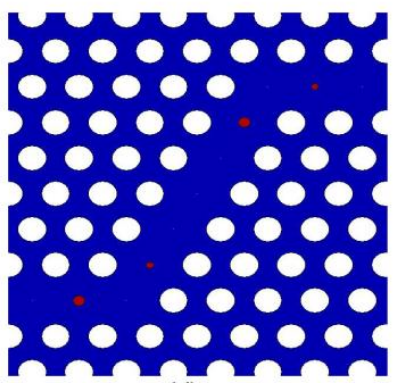

(d)

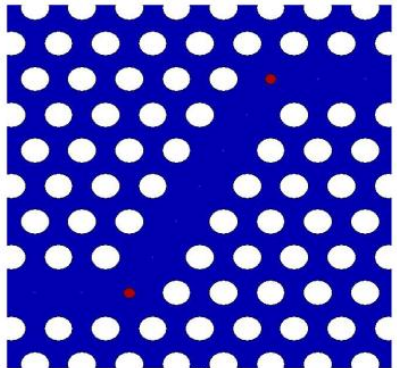

(b)

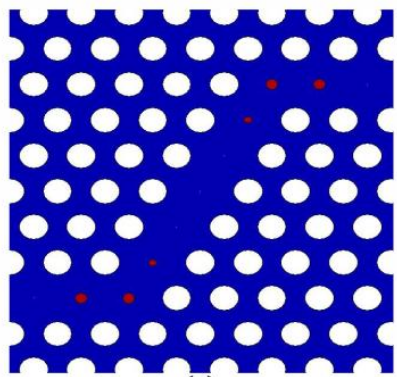

(e)

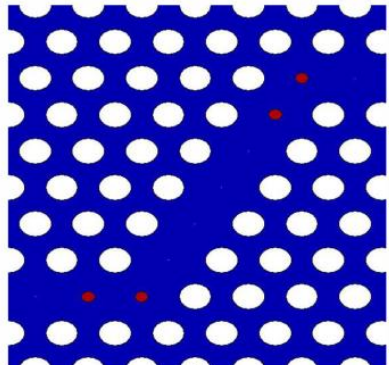

(c)

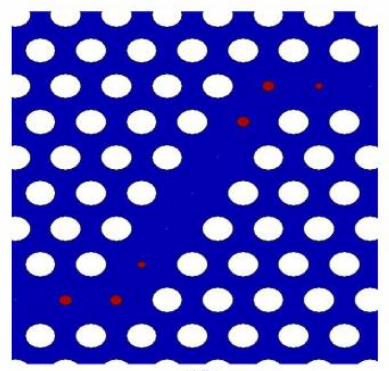

(f)

Fig. 3. (a) The standard $60^{\circ}$ double bend and (b-f) improved bends by optimisation of hole radius at the bending core.

of the bending core, the transmission window is found to be very much narrowed. In addition, the transmission drops at $1.57 \mu \mathrm{m}$, similar to the standard $60^{\circ}$ waveguide. This observation points out the importance of the center hole in the bending core. Therefore, in configuration (e), a center hole is added to configuration (d) while keeping a diagonal mirror symmetry between the input and output waveguides. When there are three holes in the bending region, the transmission window is very much broadened and flattened. In the wide range between 1.506 $\mu \mathrm{m}$ and $1.577 \mu \mathrm{m}$, transmission is over $70 \%$. The maximum transmission is found as $91 \%$ at 1.52 $\mu \mathrm{m}$. When the holes in configuration (f) are ordered in the same sequence for the two bends, the transmission window is a little enlarged at the cost of decrease in the transmission values. The maximum transmission is found as $88.9 \%$ at $1.544 \mu \mathrm{m}$. This design shows 14\% higher maximum transmission value in a wider bandwidth than the topology-optimized double bend design which involves inserting mirrors in the corners and deleting holes from external bends [15]. Configuration (e) is adopted for the whole structural analysis in Section 3.c.

Fairly well accordance between experimental and theoretical results is obtained for the transmission spectrum of the $90^{\circ}$ waveguide bend by the adjustment of the air holes in the first rows adjacent to the waveguide to $50 \mathrm{~nm}$ and the radius of second rows adjacent to the waveguide to $130 \mathrm{~nm}, 150 \mathrm{~nm}$ or $170 \mathrm{~nm}$ [16]. In our study, the optimised radii of holes at the bending core are $35 \mathrm{~nm}, 52 \mathrm{~nm}$ and $54 \mathrm{~nm}$, respectively, which can be fabricated using etching and lithography techniques. Since fabrication of holes with $35 \mathrm{~nm}$ radius $(0.08 a)$ can be challenging in real implementation, further FDTD simulations are performed under $0.02 a(8.65 \mathrm{~nm})$ variation of the $35 \mathrm{~nm}$ hole radius. The cut-off wavelength at the short wavelength side is observed to shift $0.4 \mathrm{~nm}$ under the change of hole radius, whereas the transmission curve is very less affected $( \pm 1 \%)$.

\section{3.b. Optimisation of the $\mathrm{Y}$-junction}

As shown in Fig. 5, Y-junction shows poor transmission due to modal mismatch at the junction. Efficient and symmetric power splitting of Y-junctions can be realized by using symmetric Y-junctions including a symmetric cavity or by optimising the center of the cavity [17]. However, the transmission bandwidth of the first approach is narrow and the transmission properties is very sensitive to deviations from the precise symmetry [17]. Therefore they are more suitable for on/off 


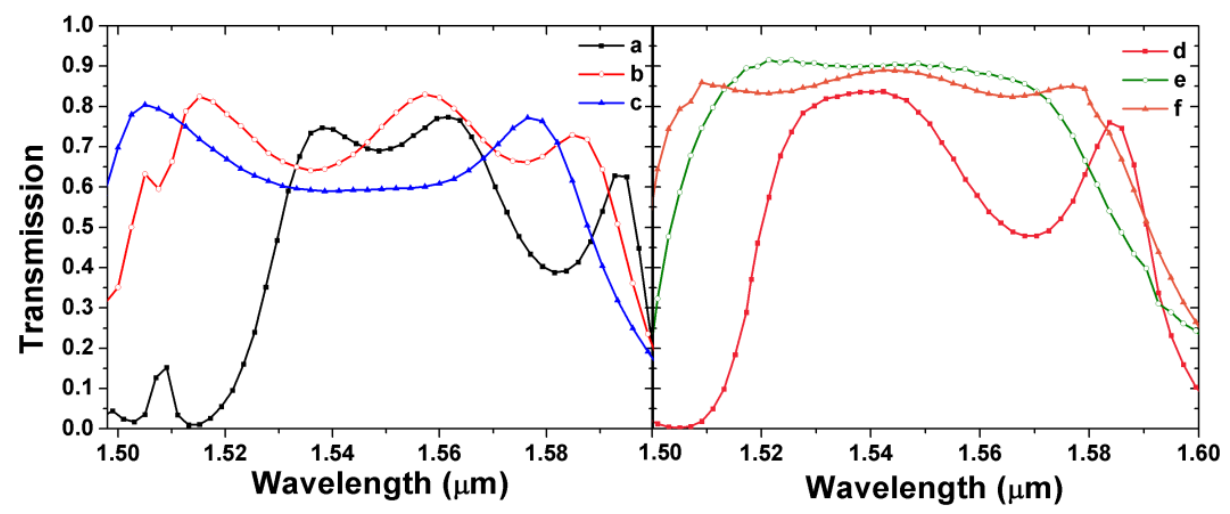

Fig. 4. Transmission spectra of the $60^{\circ}$ double bends given in Fig. 3 .

switch operation. Optimising the center of the cavity by adding holes at the junction is a rational method to obtain efficient splitting in $\mathrm{Y}$ junctions. By adding smaller holes, the optical volume is reduced, mode expansion is prevented and excitation of higher order modes is suspended. Two smaller size holes are placed at the junction and the radius values are optimised. In the optimization, the initial values and the lower and upper bounds of the two air holes are given as input parameters and an algebraic expression is defined which maximizes the total flux detected from the output sensors relative to the flux through the reference detector. The optimizations are performed automatically by changing the radii of the holes each time and outputting the flux value.

The optimised radius values are determined as $0.2111 a$ for the hole nearest to the Y-junction and $0.1522 a$ for the next hole. The optimised transmission shows more than two-fold increase in amplitude and the maximum transmission is found as 0.483 for the output- 1 at $1.53 \mu \mathrm{m}$ and 0.46 for the output-2 at $1.52 \mu \mathrm{m}$. Our transmission values at the exit ports are higher and almost the same in a wider spectral range than Ref. [15], which shows the advantage of this simple design of adding two holes at the junction to topology optimisation by deleting holes in the external bends and increasing the size of holes at the junction. Maximum power transmission of 0.4 at each exit port is experimentally demonstrated for first time in 2003 by adding two small holes in the Y-junction [6]. In our study, by performing global optimisation with a large range of radius scan, the maximum calculated transmission value is found to be higher than that in Ref. [6]. In Ref. [6], 2D FDTDoptimized air hole radii are calculated to be $0.75 r$ and $0.5 r$, where $r$ is about $100 \mathrm{~nm}$. However due to the nature of the reactive-ionetching process, the fabricated holes are found to be $0.68 r$ and $0.4 r$, respectively [6]. Nevertheless, a good agreement is obtained between model and experiment, implying very low out-of-plane loss [6]. In view of this, due to the small size of holes in the bending cores and Y-junctions, we expect a little out-of-plane loss.

\section{3.c. Optimisation of PC based directional coupler}

Directional couplers are parallel waveguides that are placed close enough so that their respective modes interact. This interaction splits the modes into even and odd symmetry supermodes. The even guided mode, which is interested in this study, splits into one even and one odd mode due to the presence of the directional coupler. The period of propagation of light periodically between through and cross waveguides is called beat length [18] and it is defined as,

$$
L_{B}=\frac{2 \pi}{\left|k_{\text {even }}-k_{\text {odd }}\right|} .
$$

If the length of the directional coupler is an odd multiple of half beat length, the field couples totally to the cross-port, whereas if the length is an integer multiple of beat length, no coupling occurs. If the length of the directional coupler is an odd multiple of a quarter beat length, equal power transmission through cross and through 


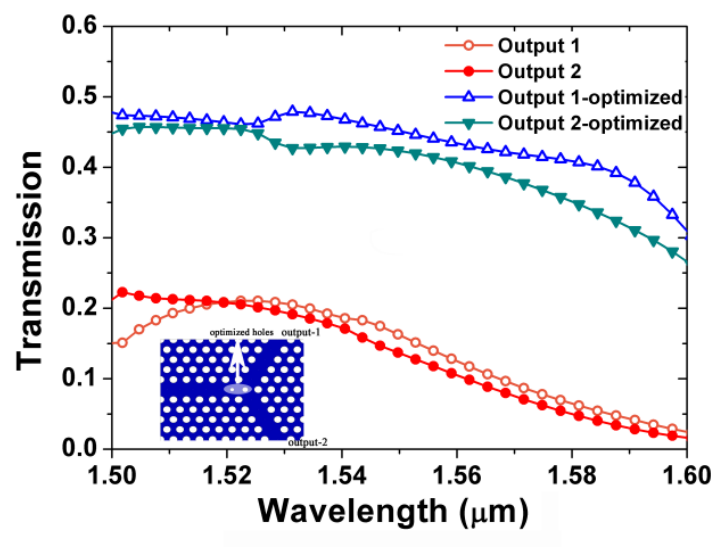

Fig. 5. Transmission spectra of the Y-junction, with and without modification.

ports can be obtained:

$$
L_{C}=\frac{(2 m+1) \pi}{2\left|k_{\text {even }}-k_{\text {odd }}\right|},
$$

with $m=1,2,3 \ldots$. In fact, the transmitted power at the cross and through ports for any arbitrary coupling length can be calculated according to the formulas [5]:

$$
\begin{gathered}
T_{\text {cross }}(L)=\sin ^{2}\left(\frac{\pi L}{L_{B}}\right), \\
T_{\text {through }}(L)=\cos ^{2}\left(\frac{\pi L}{L_{B}}\right) .
\end{gathered}
$$

The higher the wave-vector difference, the smaller the beat length is. Moreover, it is reported that as the distance between parallel waveguides increase, the coupling length increases rapidly [19]. One row of holes is remained between the parallel waveguides in order to increase the coupling coefficient and make the device as compact as possible. Fig. 6 illustrates the band diagram of the directional coupler with 0.30 a radii coupling holes. The gray shadows are the extended modes. The eveneven, even-odd, odd-odd and odd-even modes are respectively referred to as $e-e, e-O, O-o$ and $o$ $e$ modes in the graph. It is interesting to note that the e-e mode lies at a higher frequency than the e-o mode, similar to the band diagram of the directional coupler formed from triangular lattice of air holes in a $250 \mathrm{~nm}$ thick dielectric slab [20]. The $e-e$ and $e-o$ modes split at the wave-vector of $0.324(2 \pi / a)$ and at the frequency of $0.277(a / \lambda)$, which corresponds to

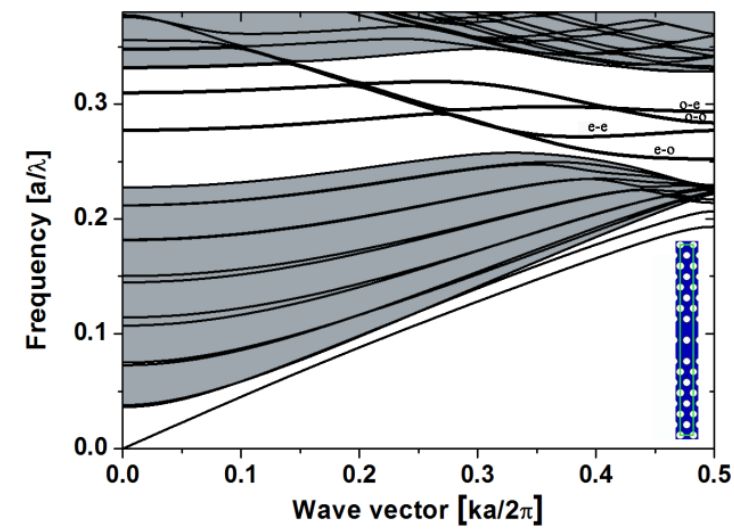

Fig. 6. The dispersion diagram of the directional coupler with $0.30 \mathrm{a}$ radius coupling holes. The supercell of the directional coupler is given in the inset.

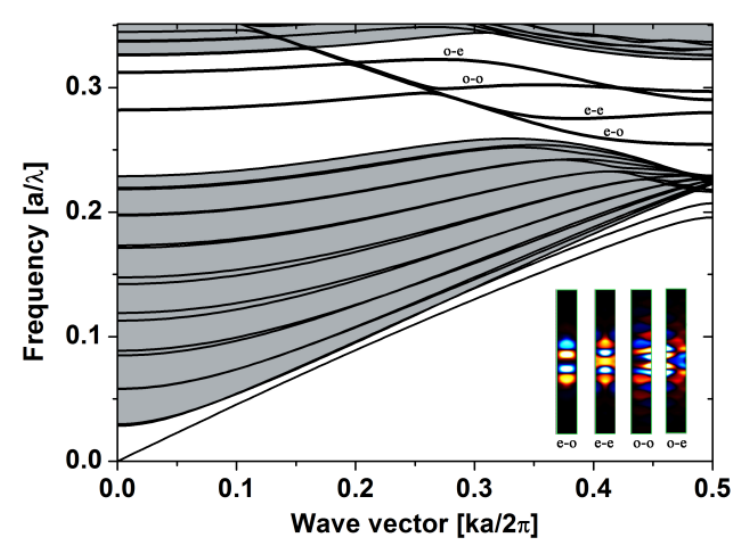

Fig. 7. The dispersion diagram of the directional coupler with $0.34 a$ radius coupling holes. The mode profiles of supermodes are given in the inset.

$1561.4 \mathrm{~nm}$. By increasing the radius of holes at the coupling region, the operating wavelength is decreased to operate the power splitter efficiently with the optimised Y-junction and double bends.

Figure 7 shows the band diagram of the directional coupler when the coupling holes are increased to $0.34 a$. Since there is node in the center of the $e$ - $o$ mode, the frequency of the e-o mode is not affected from the increase of the radius of coupling holes and the e-e mode is faced a little upward at the high wave-vector region. The $e-o$ and $e-e$ modes split at $0.29(2 \pi /$ a). It should be noted that since the coupling strength for the $\Gamma \mathrm{K}$ direction is larger than that for the $Г M$ direction, the modes of the 


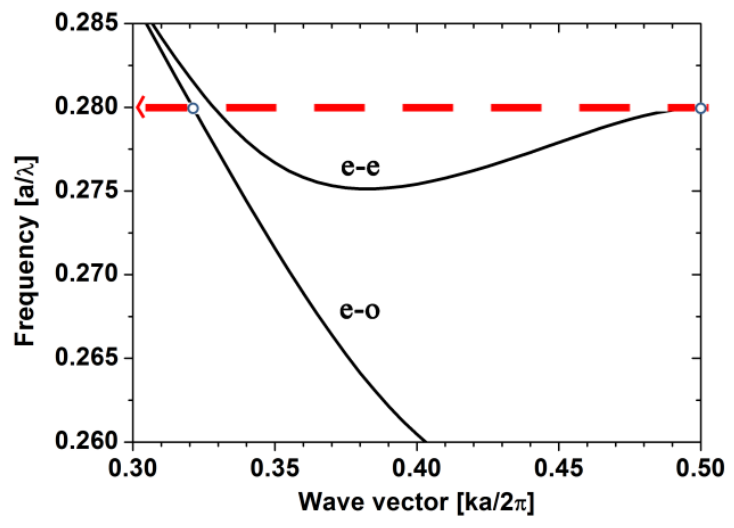

Fig. 8. The splitting of the interested $e$ - $e$ and $e$ - $o$ modes. The operating frequency is shown with a red arrow and the selected wave-vectors are marked with a circle.

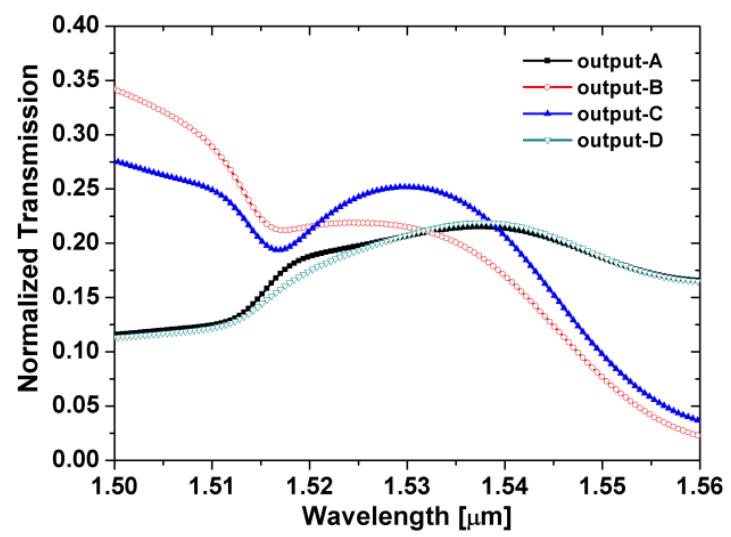

Fig. 9. Transmission spectra of output ports of the power splitter given in Fig.1.

coupler are not completely separated as for the coupled cavity PC directional couplers [21]. Figure 8 illustrates the splitting of the e-e and eo modes. The interested frequency is chosen as $0.28(a / \lambda)$ and the coupling length $L_{c}=1.397 a$ is calculated by substituting $k_{e-e}=0.3211(2 \pi / a)$ and $k_{e-o}=0.5(2 \pi / a)$ into Eq.(2). A sudden bend after coupling is not very efficient, therefore $L_{c}$ is multiplied by 5 to make it $6.985 \mathrm{a}$. Since PC is a discrete structure, $L_{c}$ is rounded off to a distance of seven lattice constant. Three lattice constant distance is left after bendings and before directional couplers to allow the field to propagate in a steady state (Fig. 1).

Transmission spectrum for all the output ports of the device is plotted in Fig. 9. At the wavelength of $1.532 \mu \mathrm{m}$, the normalized transmission values of output-A, output-B,

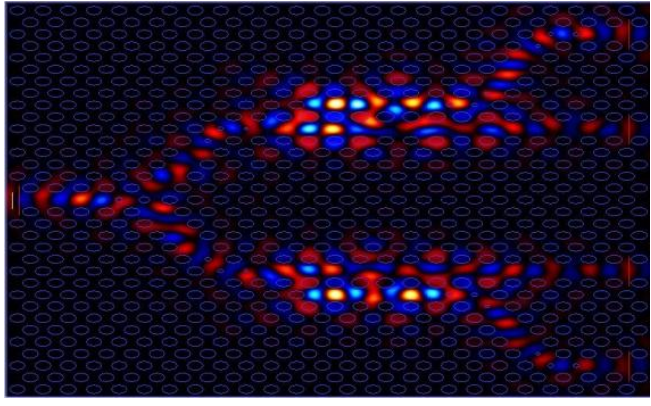

Fig. 10. FDTD simulation snap shot of Gaussian beam propagation for TE-like mode at $1.53 \mu \mathrm{m}$ central wavelength and $40 \mathrm{~nm}$ bandwidth.

output-C and output-D are 0.211, 0.211, 0.251 and 0.211 , respectively. The overall throughput efficiency is $88.5 \%$ at this wavelength. The overall throughput efficiency drops to $82.5 \%$ at $1.54 \mu \mathrm{m}$ due to inevitable reflections at the $60^{\circ}$ bends and backward reflections from the directional couplers. The device can operate for a $8 \mathrm{~nm}$ range between $1.532 \mu \mathrm{m}$ and $1.54 \mu \mathrm{m}$ with an increase in the power for output-A and $D$ and decrease in the power for output-B and $\mathrm{C}$ as the wavelength increases. Compared with the $1 \times 4$ power splitter based on directional coupling shown in Fig. 4(c) of Ref. [19], we achieve higher transmission values at the output ports and a higher overall transmission efficiency in our design based on one PC Y-junction and two PC directional couplers. The field is transported through A, B, C and D ports in an efficient way as shown in Fig. 10.

\section{Conclusions}

By optimizing the Y-junction, directional coupler and double bends, we propose a $1 \times 4$ optical power splitter device in a triangular lattice photonic crystal geometry with high transmission efficiency in the optical communication window. The optimised device can be useful for narrow-bandwidth optical switching and routing applications. In addition, by introducing a nonlinear optical material into the holes, an optical switch can be designed that outputs power at each port at the desired level. 
ÓPTICA PURA Y APLICADA. www.sedoptica.es.

\section{Acknowledgements}

We gratefully acknowledge the financial support

by Scientific Research Projects of Ankara

University (BAP) under Grant no. 12B4343011. 